Hölterhoff, Dieter; Kuklinski, Peter

\title{
Vergleichende Untersuchung der ersten Berufsschulverordnungen der 1990er Jahre in den ostdeutschen Ländern - Gemeinsamkeiten und Unterschiede
}

Seifried, Jürgen [Hrsg.]; Seeber, Susan [Hrsg.]; Ziegler, Birgit [Hrsg.]: Jahrbuch der berufs- und wirtschaftspädagogischen Forschung 2015. Opladen ; Berlin ; Toronto : Verlag Barbara Budrich 2015, S. 143-170. (Schriftenreihe der Sektion Berufs- und Wirtschaftspädagogik der Deutschen Gesellschaft für Erziehungswissenschaft (DGfE))

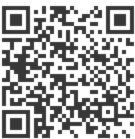

Quellenangabe/ Reference:

Hölterhoff, Dieter; Kuklinski, Peter: Vergleichende Untersuchung der ersten Berufsschulverordnungen der 1990er Jahre in den ostdeutschen Ländern - Gemeinsamkeiten und Unterschiede - In: Seifried, Jürgen [Hrsg.]; Seeber, Susan [Hrsg.]; Ziegler, Birgit [Hrsg.]: Jahrbuch der berufs- und wirtschaftspädagogischen Forschung 2015. Opladen ; Berlin ; Toronto : Verlag Barbara Budrich 2015, S. 143-170 - URN: urn:nbn:de:0111-pedocs-183501 - DOI: 10.25656/01:18350

https://nbn-resolving.org/urn:nbn:de:0111-pedocs-183501

https://doi.org/10.25656/01:18350

in Kooperation mit / in cooperation with:

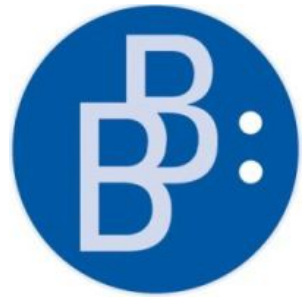

https://www.budrich.de

\section{Nutzungsbedingungen}

Gewährt wird ein nicht exklusives, nicht übertragbares, persönliches und beschränktes Recht auf Nutzung dieses Dokuments. Dieses Dokument ist ausschließlich für den persönlichen, nicht-kommerziellen Gebrauch bestimmt. Die Nutzung stellt keine Übertragung des Eigentumsrechts an diesem Dokument dar und gilt vorbehaltlich der folgenden Einschränkungen: Auf sämtlichen Kopien dieses Dokuments müssen alle Urheberrechtshinweise und sonstigen Hinweise auf gesetzlichen Schutz beibehalten werden. Sie dürfen dieses Dokument nicht in irgendeiner Weise abändern, noch dürfen Sie dieses Dokument für öffentliche oder kommerzielle Zwecke vervielfältigen, öffentlich ausstellen, aufführen, vertreiben oder anderweitig nutzen.

Mit der Verwendung dieses Dokuments erkennen Sie die Nutzungsbedingungen an.

\section{Terms of use}

We grant a non-exclusive, non-transferable, individual and limited right to using this document.

This document is solely intended for your personal, non-commercial use. Use of this document does not include any transfer of property rights and it is conditional to the following limitations: All of the copies of this documents must retain all copyright information and other information regarding legal protection. You are not allowed to alter this document in any way, to copy it for public or commercial purposes, to exhibit the document in public, to perform, distribute or otherwise use the document in public.

By using this particular document, you accept the above-stated conditions of use.

\section{Kontakt / Contact:}

peDOcs

DIPF | Leibniz-Institut für Bildungsforschung und Bildungsinformation

Informationszentrum (IZ) Bildung

E-Mail: pedocs@dipf.de

Internet: www.pedocs.de

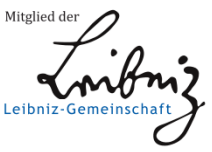




\section{Jahrbuch der berufs- und wirtschaftspädagogischen Forschung 2015}

Jürgen Seifried, Susan Seeber, Birgit Ziegler (Hrsg.)

DGfE Deutsche Gesellschatt 
Schriftenreihe der Sektion

Berufs- und Wirtschaftspädagogik

der Deutschen Gesellschaft

für Erziehungswissenschaft (DGfE) 
Jürgen Seifried

Susan Seeber

Birgit Ziegler (Hrsg.)

Jahrbuch der berufs- und

wirtschaftspädagogischen

Forschung 2015

Verlag Barbara Budrich

Opladen • Berlin • Toronto 2015 
Bibliografische Information der Deutschen Nationalbibliothek

Die Deutsche Nationalbibliothek verzeichnet diese Publikation in der Deutschen

Nationalbibliografie; detaillierte bibliografische Daten sind im Internet über

http://dnb.d-nb.de abrufbar.

Gedruckt auf säurefreiem und alterungsbeständigem Papier.

Alle Rechte vorbehalten.

C 2015 Verlag Barbara Budrich, Opladen, Berlin \& Toronto

www.budrich-verlag.de

$\begin{array}{ll}\text { ISBN } & 978-3-8474-0722-5 \text { (Paperback) } \\ \text { eISBN } & 978-3-8474-0871-0 \text { (eBook) }\end{array}$

Das Werk einschließlich aller seiner Teile ist urheberrechtlich geschützt. Jede Verwertung außerhalb der engen Grenzen des Urheberrechtsgesetzes ist ohne Zustimmung des Verlages unzulässig und strafbar. Das gilt insbesondere für Vervielfältigungen, Übersetzungen, Mikroverfilmungen und die Einspeicherung und Verarbeitung in elektronischen Systemen.

Umschlaggestaltung: Bettina Lehfeldt, Kleinmachnow - www.lehfeldtgraphic.de Typographisches Lektorat: Anja Borkam, Jena 


\section{Inhaltsverzeichnis}

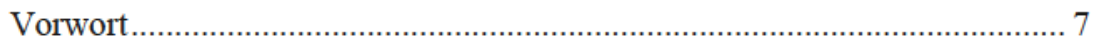

\section{Teil I: Kompetenz(facetten) und Persönlichkeitsentwicklung}

Carmela Aprea, Eveline Wuttke, Seraina Leumann, Michael Heumann Kompetenzfacetten von Financial Literacy: Sichtweisen verschiedener Akteure

Bärbel Fürstenau, Mandy Hommel, Claudia Leopold, Héctor Ponce, Mario López

Baufinanzierung nach Maß? - Aufbau von Finanzkompetenz durch

Online-Informationen 23

Sebastian Lerch

Das kompetente Selbst. Empirische Befunde zu Selbstkompetenzen in Ingenieurwesen, Pädagogik und Medizin

Raphaela Schreiber

Berufliche Identität von Zeitarbeitnehmern

\section{Teil II: Forschung zu Übergängen}

Sylvia Rahn, Thorsten Bührmann, Emanuel Hartkopf

Geplantes Verhalten im Übergangsprozess? - Berufsorientierungs- und Übergangsprozesse von Schülerinnen und Schülern einjähriger Bildungsgänge des Übergangssegments

Taiga Brahm

Resilienzförderung im Übergangssegment - Erste Ergebnisse einer Längsschnittuntersuchung.

Bernd Fitzenberger, Stefanie Licklederer, Markus Zimmermann Übergänge von der allgemeinbildenden Schule in berufliche Ausbildung und Arbeitsmarkt: Die ökonomische Perspektive 


\section{Teil III: Unterrichts-, Hochschul- und Lehrerbildungsforschung}

Nicole Kimmelmann, Katja Dippold-Schenk

Professionalisierung von Lehrpersonen in der beruflichen

Weiterbildung als didaktische Herausforderung - Erste Ergebnisse aus dem Verbundprojekt SpraSibeQ

Karl-Heinz Gerholz

Potenziale von Service Learning in den Wirtschaftswissenschaften Didaktische Konzeption und empirische Befunde aus einer Ex-anteAnalyse

Matthias Conrad, Stephan Schumann

Tablet-PCs im Wirtschaftsunterricht und die Rolle der Lehrperson

\section{Teil IV: Historische Berufsbildungsforschung}

Dieter Hölterhoff, Peter Kuklinski

Vergleichende Untersuchung der ersten Berufsschulverordnungen der 1990er Jahre in den ostdeutschen Ländern - Gemeinsamkeiten und Unterschiede

Herausgeberschaft

Autorinnen und Autoren 


\title{
Vergleichende Untersuchung der ersten Berufsschulverordnungen der 1990er Jahre in den ostdeutschen Ländern - Gemeinsamkeiten und Unterschiede
}

\author{
Dieter Hölterhoff, Peter Kuklinski
}

\section{Ausgangslage, Vorbemerkungen und Gegenstand der Untersuchung}

Die beruflichen Schulen in den ostdeutschen Ländern von 1991 bis Mitte der 1990er Jahre können für die berufsbildungswissenschaftliche historiografische Forschung als Terra incognita bezeichnet werden. In diesem Beitrag wird aus der Sicht von zwei Zeitzeugen und Beteiligten aus den Ländern Brandenburg und Sachsen anhand ausgewählter Beispiele versucht, im Rückblick und mit dem Abstand von fast 25 Jahren den Transformationsprozess der schulischen beruflichen Bildung in den ostdeutschen Ländern unter den damaligen Rahmenbedingungen zu reflektieren. Dabei ist nach Zwangsläufigkeiten zu fragen bzw. ob es „Un“"zwangsläufigkeiten im Spagat zwischen den Rahmenvereinbarungen der Ständigen Konferenz der Kultusminister der Länder in der Bundesrepublik Deutschland (KMK) und den neuen Schulgesetzen und Berufsschulverordnungen ${ }^{1}$ gab. $^{2}$ Welchen Bruch gab es zu DDR-Vorläufern, welchen Bruch mit westdeutschen Traditionen oder welche Kontinuität als einfache, unreflektierte Fortschreibung und damit Transformation der bisherigen in den Partnerländern, im Unterausschuss für Berufliche Bildung (UABBi) ${ }^{3}$ sowie den in der KMK vorherrschenden berufsschulpolitischen ${ }^{4}$ Denk- und Vorgehensweisen? Unklar bleibt hier mangels öffentlich einzusehender Dokumente - abgesehen von Brandenburg, Sachsen,

1 Rechtsförmlich korrekt handelt es sich um Verordnungen, rechtssystematisch um untergesetzliche Regelungen. Warum einzelne Länder den Begriff der „Ordnung " verwenden ist nicht klärbar.

2 An dieser Stelle kann nur versucht werden heraus $\mathrm{zu}$ arbeiten, ob und wenn ja, welche Freiräume bestanden (vgl. Hölterhoff 2014, S. 233 f.)

3 Eine Übersicht über die rechtlichen Regelungen (Anlage 1) sowie ein Verzeichnis der Abkürzungen (Anlage 2) finden sich am Ende des Beitrags.

4 Der Begriff ,Berufsschulpolitik' wird eingeführt, „um die die beruflichen Schulen betreffenden Debatten als gesonderten Diskussionsbereich zu betrachten. Gemeint ist damit nicht nur die Politik für die Berufsschule im dualen System der Berufsausbildung. Vielmehr wird der Begriff als Obergriff für alle Bildungsgänge/Schularten im beruflichen Schulsystem verwendet, um ... eine einheitliche Begrifflichkeit nutzen zu können" (Hölterhoff 2014, S. 57). 
Sachsen-Anhalt - ob es eine begleitende über die zuständigen Ministerien hinausgehende Diskussion der Entwürfe z. B. im Landesausschuss für Berufsbildung (LAB) gab bzw. zu welchen Ergebnissen diese führte. Gleichwohl gibt es Grund zur Annahme, dass die Vorgehensweise in MecklenburgVorpommern und Thüringen identisch war. ${ }^{5}$

Gegenstand der vergleichenden Untersuchung sind die Kernaussagen in den ersten Berufsschulverordnungen der neu gebildeten ostdeutschen Länder unter Berücksichtigung der Regelungen für die Berufsschule in den jeweiligen ersten Schulgesetzen. Als Bezugssystem wird die KMK-Rahmenvereinbarung über die Berufsschule aus dem Jahr 1991 herangezogen (vgl. KMK 1991). Die Untersuchung wird eingegrenzt auf den schulischen Teil der dualen Berufsausbildung (die Teilzeitberufsschule). Die weiteren Bildungsgänge, die in der Regel unter der Überschrift „Berufsschule“ gefasst werden, wie das Berufsgrundbildungsjahr (BGJ), das Berufsvorbereitungsjahr (BVJ) u. a. werden vorerst nicht betrachtet, weil sich darin eher spezifische berufsbildungspolitische sowie berufsschulpolitische Ansätze der Länder niederschlagen. ${ }^{6}$

Für die Berufsschule als Teil des dualen Berufsausbildungssystems ist anzunehmen, dass sich deren Regelungen dem „Gleichheitsminimum“ unterwerfen. Das Gleichheitsminimum ist mit dem Hohenheimer Memorandum (vgl. KMK 1998, S. 243 f.) - beschlossen auf der 252. Plenarsitzung ${ }^{7}$ am 21./ 22. Februar 1991 - für den gesamten Zuständigkeitsbereich formuliert: „Für die Gestaltung des Schulwesens ist das Prinzip der Kulturhoheit der Länder von grundlegender Bedeutung. Dabei tragen die Länder ihren föderalen Rechten und Verpflichtungen auch dadurch Rechnung, daß sie durch die Sicherung einer gemeinsamen und vergleichbaren Grundstruktur der Bildungsgänge im Schulwesen wesentliche Voraussetzungen für die erforderliche Freizügigkeit über Ländergrenzen hinweg schaffen.“

Die KMK-Regelungen (i.d.R. in Form von Rahmenvereinbarungen) dienen dem Gleichheitsminimum, durch das als äußere Klammer eine auf diese Selbstverpflichtung beruhende Sicherheit bei der gegenseitigen Anerkennung von Abschlüssen besteht (vgl. Hölterhoff 2014, S. 243f.). Somit wird nach-

5 Das lässt sich aus den nicht offiziell protokollierten Gesprächen der Verfasser mit den Kolleginnen und Kollegen, die in den jeweiligen ostdeutschen Bildungsministerien arbeiteten, folgern. Ebenso aus den halbjährlichen, ebenfalls nicht offiziell protokollierte Gesprächsrunden der ostdeutschen Abteilungsleiter beim damaligen Generalsekretär des BIBB, Hermann Schmidt, in der anstehende Fragen erörtert wurden, an denen die Verfasser teilnahmen.

6 Eine Untersuchung dieser Hintergründe würde den Rahmen dieser Untersuchung sprengen, wäre aber gleichwohl bezüglich der Frage einer eigenständigen ostdeutschen Entwicklung zu erörtern.

7 Es handelte sich um die erste Sitzung nach dem Beitritt der neuen Länder. Diese fand am historischen Ort der 1. Plenarsitzung der Konferenz der deutschen Erziehungsminister aus den siebzehn Ländern am 19./20. Februar 1948 in Stuttgart-Hohenheim statt. 
folgend analysiert, ob die Berufsschulverordnungen dem durch die KMKRahmenvereinbarung gesetzten Gleichheitsminimum entsprechen oder ob zugespitzt formuliert - diese für die Rechtsetzung in den Ländern entbehrlich war.

Die zum Untersuchungszeitpunkt gültigen Berufsschulverordnungen der Partnerländer aus den sog. „Betreuungsgemeinschaften“ werden in vorliegender Untersuchung nicht herangezogen, obwohl es aufschlussreich wäre festzustellen, ob es sich bei den neu geschaffenen Verordnungen um Blaupausen der Verordnungen der Partnerländer handelt. Eine solche vergleichende Betrachtung würde den Rahmen dieses Beitrages überschreiten. Die Betreuungsgemeinschaften ${ }^{8}$ gründeten sich in der Folge von Art 5 (Amtshilfe) des Staatsvertrages über die Währungs-, Wirtschafts- und Sozialunion (vgl. Staatsvertrag 1990): „Die Behörden der Vertragsparteien leisten sich nach Maßgabe des innerstaatlichen Rechts bei der Durchführung dieses Vertrags Amtshilfe. [...].“

\section{Zum politischen und rechtlicher Rahmen für die Transformation der nichtakademischen Berufsausbildung}

Der politische Rahmen für die Wiedervereinigung wurde durch den Vertrag über die Schaffung einer Währungs-, Wirtschafts- und Sozialunion zwischen der Bundesrepublik Deutschland und der Deutschen Demokratischen Republik (Staatsvertrag) vom 18. Mai 1990 (vgl. Staatsvertrag 1990) und durch den Staatsvertrag über die Herstellung der Einheit Deutschlands (Einigungsvertrag) vom 31. August 1990 (vgl. Einigungsvertrag 1990) gesetzt.

$\mathrm{Zu}$ dem hier behandelten Sachverhalt ist im Vertrag über die Schaffung einer Währungs-, Wirtschafts- und Sozialunion in Anlage VI (Regelungen, die in der Deutschen Demokratischen Republik im weiteren Verlauf anzustreben sind) im Punkt II (Wirtschafts- und Sozialunion) die Nr. 4 von besonderer Relevanz: „Einführung des Ordnungsrahmens und der Berufsstruktur der Bundesrepublik Deutschland im Bereich berufliche Bildung (Berufsbildungsgesetz: Erster Teil; Dritter Teil 2., 4., 6., 7. Abschnitt; Handwerksordnung: Zweiter Teil; 2., 4., 6., 7. Abschnitt, Dritter Teil; die auf diese Gesetze gestützten Ausbildungs- und Meisterprüfungsregelungen).“

Mit dem Einigungsvertrag wurde die Übernahme von Rechtsnormen der Bundesrepublik im Beitrittsgebiet geregelt. Ferner sind aus dem Einigungsvertrag insbesondere Art. 3 (Inkrafttreten des Grundgesetzes) mit der daraus

8 Brandenburg: Nordrhein-Westfalen und teilweise Berlin; Mecklenburg-Vorpommern: Bremen, Hamburg und Schleswig-Holstein; Sachsen: Baden-Württemberg und Bayern; Sachsen-Anhalt: Niedersachsen; Thüringen: Bayern, Hessen und Rheinland-Pfalz. 
resultierenden Kulturhoheit der Länder und Art. 37 Abs. 4 mit dem Ausweis der Zuständigkeit der Länder für Regelungen zur Neugestaltung des Schulwesens unter ausdrücklicher Bezugnahme auf Regelungen der KMK zur Anerkennung von Abschlüssen schulrechtlicher Art sowie weiterer einschlägiger Vereinbarungen der KMK von Bedeutung.

Mit dem Verfassungsgesetz zur Bildung von Ländern in der Deutschen Demokratischen Republik (Ländereinführungsgesetz) vom 22. Juli 1990 (vgl. Ländereinführungsgesetz 1990) wurde schließlich die Bildung der sog. „Neuen Länder“" zum 3. Oktober 1990 vorbereitet und beschlossen. Die neu gegründeten Länder traten am 25. Oktober 1991 dem Abkommen über die Geschäftsordnung des Sekretariats der KMK vom 20. Juni 1959 bei (KMK 1959). ${ }^{9}$ Dieser Akt war formal eine weitere wichtige Grundlage für die in den Ländern zu erarbeitenden Schulgesetze und Berufsschulverordnungen.

Bereits vor Bildung der neuen Länder am 3. Oktober 1990 versuchte die letzte DDR-Regierung einen neuen rechtlichen Rahmen für den vorschulischen und schulischen Bereich sowie für die Berufsausbildung (vgl. hierzu Kuklinski \& Wehrmeister 1998) zu schaffen, um den Transformationsprozess einzuleiten.

Im Juni 1990 gab der Minister für Bildung und Wissenschaft, Prof. Dr. Hans Joachim Meyer, in der Regierung de Maizière die „Anweisung zur Reifeprüfung im Schuljahr 1990/91“ heraus und verkündete Regelungen für die in Bildung begriffenen Länder: „Für die Übergangszeit bis zur Herstellung der vollen Funktionsfähigkeit der Länder und bis zum Inkrafttreten landesrechtlicher Regelungen werden unter Verantwortung des Ministeriums für Bildung und Wissenschaft vorläufige Schulaufsichtsbehörden gebildet. Die Schulaufsichtsbehörden bestehen aus den Landesschulämtern und den Schulämtern der Kreise. Bis zur Herstellung der vollen Funktionsfähigkeit der Länder und bis zum Inkrafttreten landesrechtlicher Regelungen ist das Ministerium für Bildung und Wissenschaft die oberste Schulaufsichtsbehörde. “10

Mit der Verordnung über die Aufrechterhaltung von Leistungen betrieblicher Kindergärten, polytechnischer und berufsbildenden Einrichtungen vom 6. Juni 1990 wurde der Versuch unternommen, u. a. die berufliche Ausbildung von Lehrlingen und die Berufsausbildung mit Abitur zu sichern (vgl. Verordnung 1990). Sie legte fest, dass beim Übergang der Betriebe in andere Rechtsträgerschaft durch definitive Festlegungen zu gewährleisten sei, dass diese Verpflichtungen der Betriebe übernommen oder weitergeführt werden. Zudem war vorgesehen, dass geeignete Lehrwerkstätten und andere entspre-

9 Die ostdeutschen Länder traten dem Abkommen über die Geschäftsordnung der KMK und auf diesem Weg der KMK bei. Hierfür wurden keine Staatsverträge abgeschlossen; ebenso wenig wie die Gründung der $\mathrm{KMK}$ durch Staatsverträge erfolgte. Bis zum Zeitpunkt des Beitritts gab es eher informelle Kontakte der neuen Länder zur KMK. In welcher Weise die so genannten Regierungsbeauftragten über ihre Mitarbeiterinnen und Mitarbeiter Kontakte zum Sekretariat der KMK hielten, ist nicht verifizierbar.

10 Zitiert nach Ernst 2014, S. 203. 
chende Objekte der Betriebe, die bei stark zergliederter betrieblicher Auflösung aufgrund ihrer Größe nicht in Kapitalgesellschaften überführt werden, von den bisherigen Trägern zur Nutzung als überbetriebliche Ausbildungseinrichtungen anzubieten sind.

Mit dem Gesetz über die Inkraftsetzung des Berufsbildungsgesetzes der Bundesrepublik Deutschland in der Deutschen Demokratischen Republik vom 19. Juli 1990 (vgl. IGBBiG 1990) wurden die Rechtsgrundlagen für die nichtakademische Berufsausbildung im dualen System geschaffen. Mit diesem Gesetz traten i. d. R. auch die auf dessen Grundlage erlassenen Rechtsverordnungen in Kraft. Besondere Maßgaben betrafen u. a. die Wandlung und Fortführung bestehender Lehrverhältnisse nach den geltenden Ausbildungsordnungen sowie die Verpflichtung der Betriebe, die Kapazitäten der praktischen Berufsausbildung mindestens bis zum Zeitpunkt der Erfüllung abgeschlossener Lehrverträge aufrechtzuerhalten.

Das Gesetz über Berufsschulen vom 19. Juli 1990 (vgl. Gesetz Berufsschulen DDR) regelte zudem in Anlehnung an die Landesschulgesetze der alten Länder Aufgaben, Errichtung und Finanzierung von Berufsschulen bis zum Erlass der Schulgesetze durch die neuen Länder. Die Berufsschulen wurden als öffentliche Schulen in Trägerschaft der Kreise und kreisfreien Städte definiert, die Betriebsberufsschulen zum 31. August 1990 aufgelöst. Die neuen Träger waren verpflichtet, bis zum 1. September 1990 Berufsschulen zu errichten. Das Gesetz sah vor, dass Grund und Boden sowie Gebäude, die bisher für den theoretischen Unterricht der Lehrlinge genutzt wurden, und das Inventar der Betriebsberufsschulen und kommunalen Berufsschulen mit Inkrafttreten des Gesetzes kostenlos in die Rechtsträgerschaft des örtlich zuständigen Trägers zu überführen sind. Insbesondere wurde festgelegt, dass die der theoretischen Berufsausbildung dienenden Einrichtungen einschließlich der Lehrlingswohnheime sowie der Grund und Boden nicht als der Konkursmasse zugehörig zu bewerten sind.

\section{Zur Facharbeiterausbildung in der DDR - Ausgangslage und Struktur ${ }^{11}$}

\subsection{Ausgangslage}

Die Entwicklung des ostdeutschen Berufsbildungssystems verlief zwischen 1949 und 1989 abgekoppelt und in Diskontinuität zum dualen System, allerdings ohne völlig mit den bewährten Formen der deutschen Facharbeiterausbildung zu brechen.

11 Vgl. Wehrmeister 2005, S. 70-85. 
Auf Grundlage des Befehls Nr. 40 der Sowjetischen Militäradministration wurde der Unterricht in den berufsbildenden Schulen zum 1. Oktober 1945 wieder aufgenommen. Im November 1947 trat eine gemeinsam von der Arbeitsverwaltung und der Verwaltung für Volksbildung initiierte Verordnung über die Ausbildung von Industriearbeitern in den Berufsschulen in Kraft (vgl. Verordnung 1947), die eine Anordnung über die Förderung des Berufsnachwuchses in volkseigenen Betrieben nach sich zog (vgl. Anordnung 1948). Letztere ist als offizielles Gründungsdokument der Betriebsberufsschulen zu betrachten, die offenbar in Folge des Zeitzer Jungaktivistenkongresses sowie des II. Berufspädagogischen Kongresses 1949 erlassen wurde (vgl. Akademie 1983, S. 110). Die Verordnung umfasste Rahmenbestimmungen für die betriebliche und schulische Lehrlingsausbildung und regelte die Berufsschulpflicht für Jugendliche im Alter von 14 bis 18 Jahren. Sie könnte aufgrund ihres umfassenden Inhaltes auch als „erstes deutsches Berufsausbildungsgesetz" bezeichnet werden. ${ }^{12}$ Die volkseigenen Betriebe wurden u. a. aufgefordert, die Berufsausbildung an die betriebliche Praxis zu koppeln und insbesondere den theoretischen Unterricht und die praktische Ausbildung „auf das engste“ zu vereinen. Mitte des Jahres 1949 gab es in der Sowjetischen Besatzungszone bereits 509 Betriebsberufsschulen mit 60.000 Schülerinnen und Schülern (vgl. Less 1949).

In den 1950er Jahren wurden Betriebsberufsschulen, Lehrwerkstätten und Lehrlingswohnheime zu sogenannten Lehrkombinaten zusammengeführt. Damit lag die Verantwortung für die praktische Ausbildung und den theoretischen Unterricht sowie die außerschulische Betreuung der Lehrlinge erstmals allein in den Händen eines Betriebes (vgl. Beschluss Ministerrat 1956).

Neben den Betriebsberufsschulen bestanden weiterhin kommunale Berufsschulen, die den Räten der Kreise unterstellt waren und überwiegend die Lehrlinge in Handwerks- und Dienstleistungsberufen, aber auch in landwirtschaftlichen Berufen unterrichteten. 1983 handelte es sich um rund 260 Schulen (vgl. Akademie a.a.O. S. 111), die die Aufgabe hatten, den Berufsschulunterricht durchzuführen für alle Betriebe, in denen keine Betriebsberufsschule eingerichtet war. Für Facharbeiterberufe mit geringen Lehrlingszahlen war die theoretische Berufsausbildung an ausgewählten Betriebsberufsschulen oder kommunalen Berufsschulen zentralisiert.

Die Einbindung der Berufsschule in das einheitliche Bildungs- und Erziehungssystem der DDR begann mit dem „Gesetz über die sozialistische Entwicklung des Schulwesens“ vom 2. Dezember 1959 (vgl. Gesetz Schulwesen DDR 1959). Diese Entwicklung wurde mit dem „Gesetz über das einheitliche sozialistischen Bildungssystem“ des Jahres 1965 (vgl. Gesetz Bildungswesen DDR 1965) abgeschlossen: Die Einrichtungen der Berufsausbil-

12 Das BBiG wurde in der Bundesrepublik Deutschland erst 1969 beschlossen. 
dung wurden ein grundlegender Bestandteil des Bildungssystems der DDR. Die zehnklassige allgemeinbildende polytechnische Oberschule wurde zur Regelschule und bildete den Unterbau einer i. d. R. zweijährigen Berufsausbildung. Die Berufsschulen sollten eine qualifizierte Berufsausbildung garantieren und Voraussetzungen für eine nachfolgende Fortbildung an Fachschulen sowie Möglichkeiten zur Erlangung der Hochschulzugangsberechtigung schaffen. Auch aus diesem Grunde wurde zum 1. September 1959 die Berufsausbildung mit Abitur eingerichtet. Der Erwerb der Hochschulreife in Verbindung mit dem Erlernen eines Facharbeiterberufs erfolgte innerhalb von drei Ausbildungsjahren. Der Zugang zu dieser Ausbildung wurde 1960 in einer „Direktive über die Berufsausbildung mit Abitur“ festgelegt. (vgl. Direktive 1960) Danach sollten bis $196520 \%$ der Abgänger der 10. Klassen eine Berufsausbildung mit Abitur aufnehmen können. Am Ende dieser Entwicklung wird plakativ proklamiert (vgl. Verordnung 1985): „Die Einrichtungen der Berufsbildung sind staatliche Bildungseinrichtungen. Sie sind Bestandteil der Betriebe oder den örtlichen Räten oder wirtschaftsleitenden oder zentralen Organen unterstellt. Alle Einrichtungen der Berufsbildung unterliegen der staatlichen Anleitung und Kontrolle."

\subsection{Struktur}

Der strukturelle Wandel der Berufsausbildung in der DDR vom handwerklich dominierten zu einem zentral gesteuerten und industriebetrieblich geprägten Ausbildungssystem spiegelte sich $u$. a. in der Zahl der Berufsschulen und den Schülerzahlen wider. In den ersten Nachkriegsjahren wurde die Mehrheit des Facharbeiternachwuchses in privaten Handwerksbetrieben ausgebildet (1947 ca. 80 \%).Während sich in den sechziger Jahren die Schülerzahlen an den Betriebsberufsschulen und an kommunalen Berufsschulen in etwa die Waage hielten, war in den folgenden Jahrzehnten ein deutlicher Rückgang des Anteils der kommunalen Berufsschulen an der theoretischen Ausbildung von Facharbeitern zu verzeichnen. In den 80er Jahren erhielten ca. $68 \%$ aller Lehrlinge ihre theoretische Ausbildung an Betriebsberufsschulen, ca. $32 \%$ an kommunalen Berufsschulen. Im Ergebnis der Konzentration der Lehrlingsausbildung in der Industrie erhöhte sich der Anteil der Betriebsberufsschulen an der Gesamtheit der Berufsschulen in der DDR auf $75 \%$.

Die Ausbildungsberufe wurden in der seit 1968 fortgeschriebenen ,Systematik der Ausbildungsberufe" geführt (vgl. Verordnung 1984). Danach war in der DDR zuletzt eine Ausbildung in insgesamt 374 Facharbeiterberufen möglich. ${ }^{13}$ Bei 284 Berufen war der erfolgreiche Abschluss der 10. Klasse Voraussetzung für die Aufnahme in ein Ausbildungsverhältnis. Im Rahmen

13 Damit hatte die kleinere DDR nahezu die gleiche Anzahl an Berufen wie die Bundesrepublik Deutschland. 
des Bildungsangebotes bildeten 94 Grundberufe den Regelfall der beruflichen Ausbildung. Per Definition handelte es sich dabei um Ausbildungsberufe, die im Gegensatz zum Monoberuf durch eine umfassende berufliche Grundlagenbildung im Rahmen der Berufsausbildung die Spezialisierung in insgesamt 376 Richtungen ermöglichten. ${ }^{14}$

Die Ausbildungsunterlagen enthielten Angaben zum Beruf, Rahmenstundentafeln, Hinweise zur Ausbildungsorganisation, Lehrpläne für den theoretischen sowie den berufspraktischen Unterricht, Festlegungen zur Prüfung sowie didaktisch-methodische Hinweise. ${ }^{15}$ Die Verantwortlichkeit für die berufsspezifischen Lehrplaninhalte ergab sich unmittelbar aus der Zuordnung der Facharbeiterberufe in die Zuständigkeit verschiedener Stellen: Ca. 55 \% der Facharbeiterberufe waren Kombinaten und Betrieben zugeordnet, die in diesen Berufen hauptsächlich ausbildeten. Weitere Verantwortlichkeiten lagen bei übergeordneten staatlichen Verwaltungen, wissenschaftlichen Einrichtungen oder Fachministerien. ${ }^{16}$ In allen Lehrplänen fanden die politischideologischen Zielstellungen der DDR-Berufsausbildung ihren sichtbaren Niederschlag. Unabhängig von ideologisch überformten Bildungs- und Erziehungszielen, die mehr ein Postulat sozialistischer Bildungspolitik denn unmittelbare Handlungsanleitung bildeten, gelang es in der DDR, für die berufliche Ausbildung Strukturen zu schaffen, die der Wirtschaft den Facharbeiternachwuchs sicherten. Der Betriebsberufsschule kam eine Schlüsselfunktion zu. Im Idealfall war sie eingebunden in größere betriebliche Struktureinheiten, die so genannten Betriebsschulen oder Betriebsakademien, die über Einrichtungen der theoretischen und praktischen Lehrlingsausbildung, der Weiterbildung der Mitarbeiter und für den polytechnischen Unterricht der Schüler in den Klassenstufen 7 bis 10 verfügten.

14 Hinter diesem Ansatz stand das Leitbild des „disponiblen Facharbeitersc": Ein breites Fundament an beruflichem Grundlagenwissen sollte die Voraussetzung zur flexiblen Anpassung an technische und ökonomische Veränderungen schaffen.

15 In diesem Zusammenhang kann durchaus von einem Versuch zur Schaffung „Integrierter

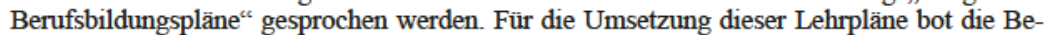
triebsberufsschule auf Grund der räumlichen Nähe zwischen Schule und betrieblicher Lehrwerkstatt günstige Bedingungen.

16 Die Lehrpläne für Staatsbürgerkunde, Sport und Betriebsökonomik wurden vom Ministerium für Volksbildung, die Lehrpläne für die technischen Grundlagenfächer vom Staatssekretariat für Berufsbildung herausgegeben. 


\section{Berufsschulen in den ostdeutschen Ländern}

\subsection{Rahmenvereinbarung der KMK über die Berufsschule}

Die Rahmenvereinbarung der KMK trifft für den schulischen Teil der Berufsausbildung nach $\mathrm{BBiG} / \mathrm{HwO}$ Aussagen über Aufgaben, Ziele, Gliederung und Organisation, Dauer und (Berufs-)Schulpflicht, Fachklassen, Unterrichtsumfang, Lehrpläne und Stundentafeln, Abschlüsse und Zeugnisse sowie über weitere schulische Berechtigungen, die an der Berufsschule erworben werden können. Weitere Bildungsgänge, die an der Schulart ${ }^{17}$ Berufsschule geführt werden (z. B. BGJ und BVJ) werden in anderen Rahmenvereinbarungen geregelt.

Die KMK-Rahmenvereinbarung über die Berufsschule wurde erstmalig und bisher nicht verändert am 15.03.1991 von der KMK nach über zehnjähriger Arbeit beschlossen. ${ }^{18}$ Die KMK hatte 1979 im Rahmen eines „Arbeitskataloges mit Prioritäten für Abstimmungen zur Sicherung notwendiger Einheitlichkeiten im Bildungswesen unter den Ländern" dem Unterausschuss für Berufliche Bildung (UABBi) den Auftrag der Erarbeitung einer Rahmenvereinbarung Berufsschule erteilt, in der alle notwendigen Sachverhalte, wie $\mathrm{z}$. B. Unterrichtsumfang, Unterrichtsorganisation etc. berücksichtigt werden sollten. Da zunächst keine Einigung erreicht werden konnte, ruhte die Arbeit für fünf Jahre, der Arbeitsauftrag wurde ohne entsprechende Beschlussgrundlage durch den UABBi mehrfach verändert und schließlich nach abschlieBender intensiver Diskussion in der Amtschefskonferenz ${ }^{19}$ dem KMKPlenum 1991 zur abschließenden Beschlussfassung vorgelegt. Vor Gründung

17 Die „KMK-Vereinbarung über die Schularten und Bildungsgänge im Sekundarbereich $\mathrm{I}^{\text {c }}$ (Stand 12.12.2013, vgl. KMK 2013) verwendet generell den Begriff Schularten (vgl. ebd. S. 4). In der Fußnote I wurde wegen Nordrhein-Westfalen, so die Auskunft des Sekretariats formuliert: "In einigen Ländern werden auch die Begriffe "Schulformen" oder „Schulgattungen" verwendet." (a.a.O.).

18 Die Angaben sind aus Unterlagen entnommen, die den Verfassern vorliegen. Nach Redaktionsschluss für diesen Beitrag wurde am 12. März 2015 die zzt. gültige Rahmenvereinbarung über die Berufsschule von der KMK beschlossen (http://www.kmk.org/fileadmin/veroeffentlichungen beschluesse/2015/2015 03 12-RV-Berufsschule.pdf) Diese fasst die Rahmenvereinbarungen über die Berufsschule vom 15.03.1991, die Vereinbarung über den Abschluss der Berufsschule vom 04.12.1997, die Empfehlung zum Einbringen der in der Berufsschule erbrachten Leistungen in das Kammerzeugnis vom 10.05.2007, die Bund-LänderVereinbarung zur Teilnahme von Berufsschülern oder Berufsschülerinnen an Austauschmaßnahmen mit dem Ausland vom 08.06.1999, die Empfehlung zur Ausgestaltung der beruflichen Grundbildung in Berufsfachschulen vom 14.10.1977 sowie Rahmenvereinbarung über das Berufsgrundbildungsjahr vom 19.05.1978 zusammen. Der Auftrag dazu erging 2007.

19 Die Amtschefskonferenz besteht aus den jeweiligen Staatsekretärinnen und Staatsrätinnen (letztere in Bremen und Hamburg), die unterhalb der MinisterInnen bzw. SenatorInnen die in den jeweiligen, die KMK bildenden Ministerien die Amtsgeschäfte leiten. 
der ostdeutschen Länder gab es ein Seminar des UABBi in Caputh in dem folgende Information gegeben wurde: „Nach langen und intensiven Beratungen hat der Unterausschuß für Berufliche Bildung nun auch die Arbeit an dem Entwurf einer Vereinbarung über die Berufsschule beschlossen." Mit dem Beschluß der „Rahmenvereinbarung über die Berufsschule“ wird dann die Kultusministerkonferenz den Schlußstein in den Gesamtrahmen der Regelungen zum beruflichen Schulwesen eingefügt haben" (KMK 1990). ${ }^{20}$

\section{2 Übersicht über die ersten schulrechtlichen Regelungen - Gemeinsamkeiten und Unterschiede}

In Tabelle 1 werden jeweils Titel, Datum des Inkrafttretens und Quellenangabe der ersten Schulgesetze und ersten Berufsschulverordnungen der neugegründeten Länder genannt. Für die beruflichen Schulen sind ausgewählte Regelungen aus den Schulgesetzen gegenüber gestellt.

Tab. 1: Erste Schulgesetze, ausgewählte Regelungen zu den beruflichen Schulen und zur Berufsschule in diesen Schulgesetzen sowie erste Verordnungen für die Berufsschule in den neuen Ländern

\begin{tabular}{|c|c|c|c|c|c|}
\hline Land & BB & MV & SN & ST & TH \\
\hline $\begin{array}{l}\text { Erste } \\
\text { Schul- } \\
\text { gesetze }\end{array}$ & $\begin{array}{l}\text { Erstes Schul- } \\
\text { reformgesetz- } \\
\text { 1. SRG vom } 25 . \\
\text { Mai } 1991 . \\
\text { GVBB S. } 116\end{array}$ & $\begin{array}{l}\text { Erstes Schul- } \\
\text { reformgesetz } \\
\text { (SRG). GVB1 } \\
\text { vom 29.04.1991 }\end{array}$ & $\begin{array}{l}\text { Schulgesetz für } \\
\text { den Freistaat } \\
\text { Sachsen } \\
\text { (SchulG) vom } 3 . \\
\text { Juli } 1991 \text {. GVB1. } \\
\text { S. } 213\end{array}$ & $\begin{array}{l}\text { Schulreform- } \\
\text { gesetz fuir das } \\
\text { Land Sachsen- } \\
\text { Anhalt (Vor- } \\
\text { schaltgesetz) } \\
\text { vom 11.07. } \\
1991 \text {. GVB1. } \\
\text { LSA Nr. 17/ } \\
\text { 1991, ausgege- } \\
\text { ben am } \\
17.07 .1991\end{array}$ & $\begin{array}{l}\text { Vorläufiges } \\
\text { Bildungsgesetz } \\
\text { (VBiG) vom } \\
\text { 25. März } 1991 . \\
\text { GVB1 TH Nr. } \\
\text { 5, S. } 61\end{array}$ \\
\hline $\begin{array}{l}\text { Gliederung } \\
\text { der berufs- } \\
\text { bildenden } \\
\text { Schulen/ } \\
\text { Schularten }\end{array}$ & $\begin{array}{l}\text { Berufsschule } \\
\text { Berufsfach- } \\
\text { schule BG zur } \\
\text { Erlangung der } \\
\text { allgemeinen } \\
\text { Hochschulreife } \\
\text { BG zur Erlan- } \\
\text { gung der Fach- } \\
\text { hochschulreife } \\
\text { Berufsqualifi- } \\
\text { zierende und } \\
\text { doppelqualifi- } \\
\text { zierende BG } \\
\text { (§ } 5 \text { Abs. } 41 \text {. } \\
\text { SRG) }\end{array}$ & $\begin{array}{l}\text { Berufsschule } \\
\text { Berufsfach- } \\
\text { schule } \\
\text { Fachoberschule } \\
\text { Fachgymnasium } \\
\text { Fachschule } \\
\text { (§ } 1 \text { Abs. 2 Nr. } 3 \\
\text { SRG) }\end{array}$ & $\begin{array}{l}\text { Berufsschule } \\
\text { Berufsfach- } \\
\text { schule } \\
\text { Fachschule } \\
\text { Fachoberschule } \\
\text { Berufliches } \\
\text { Gymnasium } \\
\text { (§4 Abs. } 1 \text { Nr. } 2 \\
\text { SchulG) }\end{array}$ & $\begin{array}{l}\text { Berufsschule } \\
\text { Berufsfach- } \\
\text { schule } \\
\text { Berufsaufbau- } \\
\text { schule } \\
\text { Fachschule } \\
\text { Fachoberschule } \\
\text { Fachgymnasium } \\
\text { (§ } 3 \text { Abs. 2 Nr. 2 } \\
\text { Vorschaltgesetz) }\end{array}$ & $\begin{array}{l}\text { Berufsschule } \\
\text { Berufsfach- } \\
\text { schule } \\
\text { Berufsauf- } \\
\text { bauschule } \\
\text { Fachober- } \\
\text { schule } \\
\text { Berufliches } \\
\text { Gymnasium } \\
\text { Fachschule } \\
\text { (§5 Abs. 1 } \\
\text { VBiG) }\end{array}$ \\
\hline
\end{tabular}

20 An dem Seminar des UABBi „Berufliches Schulwesen in der Zuständigkeit der Länder" am 6./7. September 1990 nahm einer der Verfasser teil. Die unveröffentlichte, nicht durchgehend paginierte Dokumentation liegt vor. 
Tab. 1: Erste Schulgesetze, ausgewählte Regelungen zu den beruflichen Schulen und zur Berufsschule in diesen Schulgesetzen sowie erste Verordnungen für die Berufsschule in den neuen Ländern (Fortsetzung)

\begin{tabular}{|c|c|c|c|c|c|}
\hline Land & BB & MV & SN & ST & TH \\
\hline $\begin{array}{l}\text { Organisa- } \\
\text { tionsform }\end{array}$ & $\begin{array}{l}\text { Die Schulen der } \\
\text { Sekundarstufe II } \\
\text { werden i. d. R } \\
\text { organisatorisch } \\
\text { in Oberstufen- } \\
\text { zentren (OSZ) } \\
\text { gefuihrt. } \\
\text { (§5 Abs. } 41 . \\
\text { SRG) }\end{array}$ & $\begin{array}{l}\text { Die Schularten } \\
\text { sind im Gebiet } \\
\text { eines Schul- } \\
\text { trägers i.d. R. } \\
\text { organisatorisch } \\
\text { zu einer Schule } \\
\text { zusammen- } \\
\text { gefasst. } \\
\text { (§ } 5 \text { Abs. } 3 \\
\text { SRG) }\end{array}$ & k. A. & k. A. & k. A. \\
\hline $\begin{array}{l}\text { Aufgabe/ } \\
\text { Ziel der } \\
\text { Berufs- } \\
\text { schule }\end{array}$ & $\begin{array}{l}\text { Die Berufs- } \\
\text { schule vermittelt } \\
\text { fachliche Kennt- } \\
\text { nisse und Fähig- } \\
\text { keiten und er- } \\
\text { weitert die } \\
\text { allgemeine } \\
\text { Bildung. } \\
\text { (§12 Abs. } 11 . \\
\text { SRG) }\end{array}$ & $\begin{array}{l}\text { Die Berufs- } \\
\text { schule vermittelt } \\
\text { Berufsschul- } \\
\text { pflichtigen } \\
\text { Kenntnisse, } \\
\text { Fähigkeiten und } \\
\text { Fertigkeiten, die } \\
\text { für einen Beruf } \\
\text { erforderlich sind } \\
\text { und erweitert die } \\
\text { allgemeine } \\
\text { Bildung. } \\
\text { (§ 3 Abs. 1 } \\
\text { SRG) }\end{array}$ & $\begin{array}{l}\text { Die Berufs- } \\
\text { schule hat die } \\
\text { Aufgabe, im } \\
\text { Rahmen der } \\
\text { Berufsausbil- } \\
\text { dung oder Be- } \\
\text { rufsausübung } \\
\text { vor allem fach- } \\
\text { theoretische } \\
\text { Kenntnisse zu } \\
\text { vermitteln und } \\
\text { die allgemeine } \\
\text { Bildung zu } \\
\text { vertiefen und zu } \\
\text { erweitern. } \\
\text { (§ } 8 \mathrm{Abs} \text {. } \\
\text { SchulG) }\end{array}$ & $\begin{array}{l}\text { Die Berufs- } \\
\text { schule vermittelt } \\
\text { ihren Schülern } \\
\text { fachliche und } \\
\text { allgemeine Bil- } \\
\text { dung unter Be- } \\
\text { rücksichtigung } \\
\text { der Anforde- } \\
\text { rungen der Be- } \\
\text { rufsausbildung } \\
\text { und der Berufs- } \\
\text { ausübung. Sie } \\
\text { hat insbesondere } \\
\text { im Rahmen des } \\
\text { dualen Systems } \\
\text { der Berufsaus- } \\
\text { bildung die Auf- } \\
\text { gabe, die Schü- } \\
\text { ler beruflich zu } \\
\text { bilden und zu er- } \\
\text { ziehen. } \\
\text { ( } 9 \text { Abs. } 1 . \text { Vor- } \\
\text { schaltgesetz) }\end{array}$ & $\begin{array}{l}\text { Die Berufs- } \\
\text { schule führt in } \\
\text { Teilzeitunter- } \\
\text { richt im Rah- } \\
\text { men der dua- } \\
\text { len Berufsaus- } \\
\text { bildung zu be- } \\
\text { ruflichen Qua- } \\
\text { lifikationen. } \\
\text { (§5 Abs. } 2 \\
\text { VBiG) }\end{array}$ \\
\hline $\begin{array}{l}\text { Erste Ver- } \\
\text { ordnungen } \\
\text { für die Be- } \\
\text { rufsschule }\end{array}$ & $\begin{array}{l}\text { Vorläufige } \\
\text { Berufsschul- } \\
\text { ordnung im } \\
\text { Land Branden- } \\
\text { burg } \\
\text { (BSO) vom } 7 . \\
\text { Januar } 1992 . \\
\text { GVB1. II Nr. } 7 \\
\text { vom 18. Febr. } \\
\text { 1992, S. 40-45 }\end{array}$ & $\begin{array}{l}\text { Verordnung über } \\
\text { die Berufsschule } \\
\text { in Mecklenburg- } \\
\text { Vorpommern } \\
\text { (BSVO) vom } 5 . \\
\text { Juli 1996. } \\
\text { GVOB1 } 1996 \text { M- } \\
\text { V S. } 480\end{array}$ & $\begin{array}{l}\text { Verordnung des } \\
\text { Sächsischen } \\
\text { Staatsministe- } \\
\text { riums für Kultus } \\
\text { (SMK) über die } \\
\text { Berufsschule } \\
\text { (Schulordnung } \\
\text { Berufsschule - } \\
\text { BSO) vom 11. } \\
\text { März 1994. } \\
\text { GVB1. S. } 477\end{array}$ & $\begin{array}{l}\text { Verordnung über } \\
\text { Berufs-bildende } \\
\text { Schulen } \\
\text { (BbSVO) } \\
\text { 11. Juni } 1992 \text {. } \\
\text { GVB1 LSA Nr. } \\
\text { 24/1992 vom } 17 . \\
\text { Juni } 1992, \mathrm{~S} \text {. } \\
441-463\end{array}$ & $\begin{array}{l}\text { Vorläufige } \\
\text { Thüringer Ver- } \\
\text { ordnung über } \\
\text { das berufs- } \\
\text { bildende } \\
\text { Schulwesen } \\
\text { (VTVbSch) } \\
\text { vom 30. Okt. } \\
\text { 1991. GAB1. } \\
7 / 1991 \text { S. } 294- \\
298\end{array}$ \\
\hline
\end{tabular}

In allen ostdeutschen Ländern wurden zwischen März und Juli 1991 eigene Schulgesetze erarbeitet und in Kraft gesetzt, um schnellstmöglich eine Rechtsgrundlage für das neu zu schaffende Schulsystem zu haben. Den Ge-

21 Die Berufsschule im dualen Ausbildungssystem ist in dieser übergreifenden Verordnung in Teil 1 Abschnitte 5 und 6, sowie Teil 2 Abschnitt 1 geregelt. 
setzgebern sowie den zuständigen Ministerien war dabei durchaus bewusst, dass eine rein formale Übernahme oder zu starke Anlehnung an den entsprechenden Rechtsrahmen der Partnerländer möglicherweise die Spezifika der ostdeutschen Länder unzureichend berücksichtigen würde. Die beruflichen Schulen waren zudem überwiegend als Betriebsberufsschulen institutionalisiert (s. Abschnitt 3.2) und die gesamte nichtakademische Berufsausbildung stringent auf planwirtschaftliche Vorgaben ausgerichtet. Mit dem wirtschaftlichen Umbruch in Ostdeutschland veränderte sich der Arbeits- und Ausbildungsmarkt bedingt durch die Privatisierung und die Stilllegung von Betrieben durch die Treuhand ${ }^{22}$ grundsätzlich und führte zu dem bekannten Mangel an Ausbildungsplätzen. Die nichtakademische Berufsausbildung und das berufliche Schulsystem konnten nicht mehr in den bisherigen Organisationsformen arbeiten. Entsprechend vorsichtig wurden zunächst in Thüringen ein „Vorläufiges Bildungsgesetz“, in Sachsen-Anhalt ein „Vorschaltgesetz“ sowie in Mecklenburg-Vorpommern und Brandenburg jeweils ein „Erstes Schulreformgesetz" beschlossen. Sachsen hat sich gleich für ein "Schulgesetz" entschieden, möglicherweise um eine weitere Diskussion um die „Mittelschule" und das achtjährige Gymnasium zu vermeiden. In diesen Schulgesetzen werden allerdings bei den Regelungen für die beruflichen Schulen kaum strukturelle Unterschiede zwischen den einzelnen Ländern sichtbar (vgl. Tab. 1).

Für die berufsqualifizierenden Bildungsgänge werden in allen Ländern die Berufsschule, die Berufsfachschule sowie die Fachschule eingerichtet. Die Berufsfachschule kann sowohl berufsqualifizierende als auch berufsausbildungsvorbereitende Bildungsgänge umfassen. Für die Studienqualifizierung etablieren alle Länder auch im Bereich der beruflichen Schulen Schularten zum Erwerb der Fachhochschulreife sowie der allgemeinen Hochschulreife, die sich nur marginal in der Benennung unterscheiden: An der Fachoberschule kann die Fachhochschulreife, am Beruflichen Gymnasium (Sachsen und Thüringen) oder Fachgymnasium (Mecklenburg-Vorpommern und Sachsen-Anhalt) die allgemeine Hochschulreife erworben werden. Im Brandenburgischen Ersten Schulreformgesetz wurde in $\S 5$ Abs. 4 die Schulstruktur für die Sekundarstufe II mit der Berufsschule, der Berufsfachschule, den Bildungsgängen ${ }^{23}$ zur Erlangung der allgemeinen Hochschulreife (insbesondere die gymnasiale Oberstufe), den Bildungsgängen zur Erlangung der Fachhochschulreife sowie berufsqualifizierenden und doppelqualifizierenden Bildungsgängen definiert. „Die Schulen der Sekundarstufe II werden in der Regel in Oberstufenzentren (OSZ) geführt und organisatorisch so strukturiert, dass ein möglichst breites Angebot schulischer und beruflicher Bildungsgänge vorhanden ist" (Jehkul, Belkner \& Allmann 1991, S. 35). Als Beson-

22 Vgl. hierzu Lüdtke \& Quast (1992).

23 Der Begriff des Bildungsgangs wird nur in der Sekundarstufe II verwendet. Damit soll die zu vermittelnde Qualifikation beschrieben werden. 
derheit ist hervorzuheben, dass nicht die Schulabschlüsse, sondern die dahin führenden Bildungsgänge genannt wurden, ohne sie einer Schulform zuzuweisen. (ebd. S. 39) Die spezifische Definition für die Berufsschule erfolgt im umfassenden Sinn in $\S 12$ sowie nachfolgend für die Berufsfachschule, Fachoberschule und Fachschule in den $\S \S 13$ bis 15 (ebd. S. 63-66). Die gymnasiale Oberstufe wird in $\S 11$ beschrieben und kann mit berufsqualifizierenden Bildungsgängen zu doppelqualifizierenden Bildungsgängen verbunden werden, sowie generell an berufsbildenden Schulen mit einer beruflichen Qualifikation gekoppelt werden (ebd. S. 60).

In Brandenburg konnte und kann an den OSZ die berufsbezogene ${ }^{24}$ Hochschulreife in Wirtschaft und im gewerblich-technischen Bereich sowie die allgemeine Hochschulreife erworben werden. ${ }^{25}$

Sachsen-Anhalt und Thüringen führen zusätzlich die Berufsaufbauschule zum Erwerb des Realschulabschlusses ein, obwohl diese Schulart möglicherweise gar nicht notwendig war, da die Mehrzahl der Schülerinnen und Schüler, die in Ostdeutschland eine Berufsausbildung aufnehmen wollte, über den Abschluss der zehnklassigen polytechnischen Oberschule verfügte.

Die Regelung im brandenburgischen Schulreformgesetz zur Doppelqualifizierung kann als Alleinstellungsmerkmal gewertet werden. Hier wurde bewusst ein rechtlicher Rahmen geschaffen, um eine erfolgreiche Entwicklung in Ostdeutschland fortzusetzen. Zur organisatorischen Gestaltung des beruflichen Schulwesens gibt es im brandenburgischen Schulreformgesetz in $\S 5$ Abs. 4 die Festlegung, dass die Schulen der Sekundarstufe II i. d. R. in OSZ zu führen sind. Damit wurde die berufsschulpolitische Diskussion der 1980er Jahre zur Gestaltung einer einheitlichen (beruflichen) Schulform in der Sekundarstufe II aufgegriffen. (vgl. Hölterhoff 2014, S. 85 f., S. 138 f.) In Mecklenburg-Vorpommern sind die beruflichen Schularten im Gebiet eines Schulträgers zu einer Schule zusammenzufassen. In Sachsen werden die entsprechenden Schularten von Anfang an zwar organisatorisch in Beruflichen Schulzentren geführt, eine entsprechende Regelung wurde aber erst bei der Schulgesetznovellierung im Jahre 2004 in das Schulgesetz aufgenommen. ${ }^{26}$ In den Schulgesetzen von Sachsen-Anhalt und Thüringen gibt es keine Angaben zur Organisation der beruflichen Schulen.

Die Berufsschulverordnungen der neuen Länder entstehen zwischen 1991 und 1996 (vgl. Übersicht 1). Thüringen erlässt 1991 eine Vorläufige Verordnung über das berufsbildende Schulwesen, in der Regelungen für alle beruflichen Schularten enthalten sind. Sachsen-Anhalt setzt 1992 eine Verordnung

24 Die KMK verwendet die Bezeichnung „fachgebundene Hochschulreife ${ }^{c}$.

25 Dieser Bildungsgang wurde dann eingerichtet, wenn der Schulträger den Bedarf sah. Im Zuge der Einführung des zwölfjährigen Gymnasiums war dies für Absolventen der Sekundarstufe I die Möglichkeit sich für den Bildungsgang der allgemeinen Hochschulreife zu entscheiden, wenn der berufsbezogene Bildungsgang nicht gewünscht wurde.

26 Der Schulträger soll berufsbildende Schulen in Beruflichen Schulzentren zusammenfassen. (§ 22 Abs. 3 Sächsisches Schulgesetz). 
über Berufsbildende Schulen in Kraft und bleibt bei der Vorgehensweise einer zusammenfassenden Regelung für alle beruflichen Schulen. Die übrigen ostdeutschen Länder erstellen spezifische Verordnungen für die Berufsschule. Dabei weist Brandenburg diese im Januar 1992 als vorläufige Schulordnung Berufsschule aus. Sachsen setzt 1994 eine Schulverordnung Berufsschule in Kraft, hat aber bereits 1991 eine Prüfungsverordnung für alle die beruflichen Schulen erstellt, für die die Prüfungszuständigkeit beim Land liegt.

\subsection{Vergleich der Berufsschulverordnungen mit Bezug zur Rahmenvereinbarung}

Die Rahmenvereinbarung von 1991 bezieht sich mit den Bezeichnungen und der Gliederung auf den noch heute gültigen Beschluss der KMK vom 08.12.1975 (vgl. KMK 1991), wonach Berufsschulen die Schulen für Berufsschulpflichtigen sowie Berufsschulberechtigten sind, die sich in der beruflichen Erstausbildung befinden oder in einem Arbeitsverhältnis stehen. Den Schülerinnen und Schülern sind allgemeine und fachliche Lerninhalte unter besonderer Berücksichtigung der Anforderungen der Berufsausbildung zu vermitteln. Die Unterrichtsorganisation sieht Teilzeitform an einem oder mehreren Wochentagen oder in zusammenhängenden Teilabschnitten (Blockunterricht) vor. Er soll in enger Beziehung zur Ausbildung in Betrieben einschließlich überbetrieblicher Ausbildungsstätten stehen. ${ }^{27}$ Aus dem in den 1970er und 1980er Jahren teilweise eingeführten BGJ in Vollzeitunterricht oder in kooperativer Form - bei vorliegendem Berufsausbildungsvertrag - ergibt sich eine Gliederung in Grund- und Fachstufe, ${ }^{28}$ die jedoch keine praktische Folgerung für die Unterrichtsorganisation bzw. die Rahmenlehrpläne hatte.

27 Die sich daraus ergebende Anforderung an die Lernortkooperation und die seit Jahren darüber geführte Diskussion wird hier nicht aufgegriffen.

28 Erstaunlicherweise und kaum erklärbar wird dieser Begriff in der Rahmenvereinbarung von 1991 verwandt, obwohl das BGJ zu diesem Zeitpunkt bereits keine Bedeutung mehr hat und die Rahmenlehrpläne der in den 1980er Jahren neugeordneten Metall- und Elektroberufe dies auch nicht mehr vorsahen. 
Wie sich aus verschiedenen Beschlüssen der KMK ergibt, hat man sich inhaltlich von dieser Gliederung verabschiedet, ohne jedoch die Rahmenvereinbarung über die Berufsschule neu zu fassen. ${ }^{29}$

Nachfolgend werden ausgewählte Abschnitte der Rahmenvereinbarung über die Berufsschule vorgestellt und mit Bezug darauf die Länderregelungen in den ersten z.T. als vorläufig gekennzeichneten Berufsschulverordnungen erörtert. Die Verfasser folgen aus systematischen Gründen nicht der Gliederung der Rahmenvereinbarung, sondern versuchen, die Struktur der Verordnungen der Länder zu Grunde zu legen, um Unterschiede und Gemeinsamkeiten sichtbar zu machen Allerdings ist die Reihenfolge der Regelungssachverhalte in den Verordnungen nicht einheitlich.

\subsubsection{Gliederung und Organisation der Berufsschule}

Alle Berufsschulverordnungen regeln zunächst den Geltungsbereich: Brandenburg, Mecklenburg-Vorpommern, Sachsen-Anhalt und Thüringen benennen dazu die Bildungsgänge der Berufsschule bzw. der beruflichen Schulen. In den Verordnungen der Länder Brandenburg und Mecklenburg-Vorpommern wird die Berufsschule als ,schulischer Teil der Berufsausbildung nach $\mathrm{BBiG} / \mathrm{HwO}$ " bezeichnet, Mecklenburg-Vorpommern verwendet zudem den Terminus „Teilzeitberufsschule“. Daneben werden die Bildungsgänge BGJ und BVJ sowie einige weitere spezifische Bildungsgänge aufgeführt.

Die sächsische Verordnung bezieht sich im Geltungsbereich als einzige auf die Trägerschaft und benennt hierzu nur die öffentlichen Berufsschulen. In Sachsen-Anhalt gilt die Verordnung für öffentliche Schulen und staatlich genehmigte Ersatzschulen, in Thüringen für öffentliche Schulen und Schulen in freier Trägerschaft. Alle Verordnungen, mit Ausnahme der brandenburgischen, orientieren sich an der KMK-Rahmenvereinbarung zur Gliederung der Berufsschule in Grund- und Fachstufe.

29 Vgl. dazu u. a. „Die Berufsschule - Zusammenfassende Darstellung einschlägiger Beschlüsse der KMK" vom 20.07.2007. Der Erarbeitung ging ein Auftrag zur Zusammenfassung der einschlägigen Rahmenvereinbarungen voraus (,Grundlagen und Maßnahmen zur Optimierung der Organisation des Berufsschulunterrichts - Aktualisierter Bericht der Kultusministerkonferenz ${ }^{c c}$; Beschluss der KMK vom 12.09.1997), der sich zwar auf die geltende Rahmenvereinbarung bezieht, aber diese Gliederung nicht mehr erwähnt. Vgl. hierzu auch „Handreichung für die Erarbeitung von Rahmenlehrplänen der Kultusministerkonferenz für den berufsbezogenen Unterricht in der Berufsschule und ihre Abstimmung mit Ausbildungsordnungen des Bundes für anerkannte Ausbildungsberufe ${ }^{c}$ (Veröffentlichung der KMK vom 23.09.2011), die immer wieder aktualisiert für die seit 1996 nach Lernfeldern strukturierten Rahmenlehrpläne für den berufsbezogenen Unterricht in der Berufsschule gilt. 
Abb. 1: Auszüge aus der Rahmenvereinbarung über die Berufsschule; Abschnitt 3: Gliederung und Organisation der Berufsschule

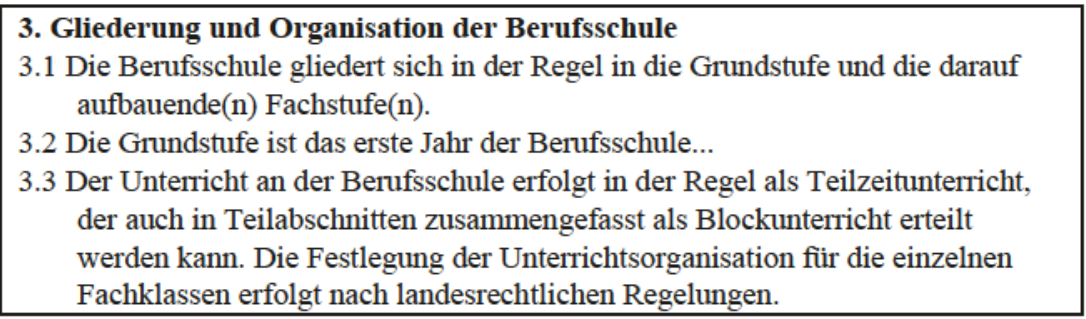

Quelle: $K M K$ (2007, S. 4f.)

Die für eine funktionierende Berufsausbildung unabdingbare Kooperation mit den Ausbildungsbetrieben sowie über- und außerbetrieblichen Ausbildungsstätten wird in den Verordnungen von Brandenburg, MecklenburgVorpommern und Sachsen berücksichtigt. Sachsen-Anhalt und Thüringen machen dazu keine Angaben. So heißt es in der brandenburgischen Verordnung, dass die Berufsschule mit allen Ausbildungspartnern und den Berufsbildungsausschüssen der zuständigen Stellen kooperiert. Mecklenburg-Vorpommern weitet dies auf die Prüfungsausschüsse aus. Sachsen formuliert allgemeiner, indem nur auf das Zusammenwirken mit den Arbeitgebern und den Arbeitnehmervertretern der Ausbildungsbetriebe sowie den Auszubildenden hingewiesen wird. Bei der Erstellung von Prüfungsaufgaben und der Durchführung der Abschlussprüfung wirkt die Berufsschule nach den gegebenen Möglichkeiten mit.

Bei den Aufnahmevoraussetzungen lassen sich gleichfalls Unterschiede erkennen: Brandenburg und Mecklenburg-Vorpommern unterscheiden nicht zwischen Berufsschulpflichtigen und Berufsschulberechtigten. Sachsen differenziert zwischen den beiden Gruppen. Berufsschulberechtigten sowie Umschülern wird der Berufsschulbesuch bei Vorliegen entsprechender schulischer Voraussetzungen eingeräumt. Sachsen-Anhalt macht keine Angaben zu den Aufnahmevoraussetzungen. Die Thüringer Verordnung enthält Regelungen für Berufsschulpflichtige; zudem sieht die Verordnung den Unterricht von Auszubildenden mit allgemeiner Hochschulreife ab der Fachstufe I vor.

\subsubsection{Dauer der Berufsschule und der Schulpflicht, Klassenbildung}

Die Berufsschulpflicht und deren Dauer werden durch die Schulgesetze der Länder bestimmt. So sind in den Berufsschulverordnungen von Mecklenburg-Vorpommern, Sachsen und Sachsen-Anhalt dazu auch keine weiteren Regelungen enthalten. Brandenburg wiederholt in der Verordnung die Regelungen des Schulgesetzes mit geringfügiger Präzisierung. Thüringen ergänzt 
in der Verordnung die Regelung des Schulgesetzes durch die Angabe, wo Berufsschulpflichtige ihre Schulpflicht erfüllen müssen: Jugendliche, die in einem Ausbildungsverhältnis stehen, besuchen die Berufsschule, die für den im Ausbildungsvertrag festgelegten Ausbildungsort zuständig ist (,Ausbildungsortprinzip"). In Brandenburg wird das „Ausbildungsortprinzip“ im Schulgesetz (§ 32) festgelegt. Sachsen schreibt in der Berufsschulverordnung (§ 9 Abs. 2) dagegen für Berufsschulpflichtige und Berufsschulberechtigte, die in einem Ausbildungsverhältnis stehen, das Wohnortprinzip vor. Allerdings kann die Schulaufsichtsbehörde zur Sicherung der Einheitlichkeit der dualen Berufsausbildung Ausnahmen zulassen.

Abb. 2: Auszüge aus der Rahmenvereinbarung über die Berufsschule; Abschnitte 4 und 5: Dauer der Berufsschule und Schulpflicht sowie Fachklassen an der Berufsschule

\section{Dauer der Berufsschule und der Schulpflicht, Klassenbildung}

4.1 Die Dauer des Bildungsgangs der Berufsschule entspricht in der dualen Berufsausbildung der Dauer des Berufsausbildungsverhältnisses. Das Berufsgrundbildungsjahr dauert ein Schuljahr.

4.3 Dauer und Umfang der (Berufs-)Schulpflicht werden auf der Grundlage der „Empfehlung zu Einzelregelungen für die (Berufs-)Schulpflicht“ (Beschluss der Kultusministerkonferenz vom 30.01.1981) durch die Länder geregelt.

4.4 Bei der Beurlaubung vom Unterricht der Berufsschule ist ein strenger Maßstab anzulegen. Einzelheiten regeln die Länder auf der Grundlage der „Empfehlung zur Beurlaubung von Berufsschülern" (Beschluss der Kultusministerkonferenz vom 30.05.1980).

\section{Fachklassen an der Berufsschule}

5.1 Die Erfuillung des Bildungsauftrags der Berufsschule erfordert die Einrichtung von Fachklassen. Der Unterricht soll grundsätzlich in Fachklassen eines Ausbildungsberufs oder verwandter Ausbildungsberufe erteilt werden.

5.2 In anerkannten Ausbildungsberufen mit einer geringen Zahl Auszubildender werden länderübergreifende Fachklassen in der Regel nach der ,Rahmenvereinbarung über die Bildung länderübergreifender Fachklassen für Schüler in anerkannten Ausbildungsberufen mit geringer Zahl Auszubildender" (Beschluss der Kultusministerkonferenz vom 26.01.1984) gebildet.

Quelle: $K M K$ (2007, S. 5f.)

Auch zur Fachklassenbildung sind die grundlegenden Regelungen in den jeweiligen Schulgesetzen erhalten. Brandenburg und Sachsen ergänzen und konkretisieren die Regelungen in ihren Berufsschulverordnungen. Im Grundsatz gilt das Fachklassenprinzip, es ist aber auch der gemeinsame Unterricht bei verwandten Berufen möglich. Sachsen-Anhalt regelt in der Berufsschulverordnung, dass Auszubildende an Fachklassen außerhalb von Sachsen-An- 
halt teilnehmen können, wenn auf Grund zu geringer Zahl von Auszubildenden keine Landesfachklasse gebildet werden kann. Auch in der Brandenburger Berufsschulverordnung wird Bezug auf länderübergreifende Fachklassen genommen. $^{30}$

\subsubsection{Stundentafeln und Lehrpläne, Leistungsfeststellung und Abschlüsse sowie weitere schulische Berechtigungen}

Die Regelungsbereiche Stundentafeln und Lehrpläne, Leistungsfeststellungen sowie Abschlüsse betreffen Sachverhalte, die nicht generell in den Berufsschulverordnungen, sondern häufig durch Verwaltungsvorschriften präzisiert wurden. Bis zur Herausgabe von Verordnungen und/oder Verwaltungsvorschriften wurden häufig auch Erlasse oder Rundschreiben ${ }^{31}$ erarbeitet.

Brandenburg, Mecklenburg-Vorpommern und Thüringen haben die Rahmenstundentafeln jeweils als Anlagen den Verordnungen beigefügt. In Sachsen wurden die Rahmenstundentafeln zunächst über Erlasse bekannt gegeben wurden, bevor 1996 dazu eine Verwaltungsvorschrift in Kraft trat. Für Sachsen-Anhalt sind keine Angaben zu den Stundentafeln in der Berufsschulverordnung enthalten.

Zum Unterrichtsumfang bei Teilzeitunterricht an einzelnen Wochentagen machen drei Länder in ihren Berufsschulverordnungen genaue Angaben: In Brandenburg und Mecklenburg-Vorpommern beträgt der Berufsschulunterricht 12, in Sachsen 13 Wochenstunden. ${ }^{32}$ In Sachsen-Anhalt und Thüringen finden sich dazu in der Berufsschulordnung keine Hinweise. In Brandenburg sowie in Sachsen-Anhalt enthält die Berufsschulverordnung zudem Regelungen für den Teilzeitunterricht in Blockform. Brandenburg hat dazu eine weitgehende Mitwirkung durch Beteiligung der Kammern und des LAB vorgesehen. $^{33}$

30 Die länderübergreifenden Fachklassen werden durch eine Rahmenvereinbarung geregelt, die jährlich mit einem hohen Arbeitsaufwand überarbeitet und mit den Spitzenorganisationen der Wirtschaft abgestimmt wird. Rahmenvereinbarung über die Bildung länderübergreifender Fachklassen für Schüler/Schülerinnen in anerkannten Ausbildungsberufen mit geringer Zahl Auszubildender (Beschluss der Kultusministerkonferenz vom 26.01.1984 i.d.F. vom 01.10.2010)

31 In den Ländern werden unterschiedliche Bezeichnungen verwendet.

32 Es wird die Angabe der KMK-Rahmenvereinbarung (,Wochenstunde ${ }^{c c}$ ) übernommen; kor-

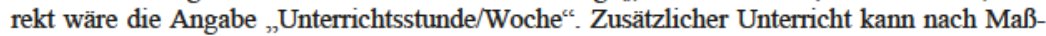
gabe der jeweiligen personellen, sächlichen und schulorganisatorischen Voraussetzungen der Schulen z. B. für Stütz- und Förderunterricht (Brandenburg, Sachsen), Religion/Philosophie (Mecklenburg-Vorpommern) und berufsspezifische Wahlfächer (Sachsen) angeboten werden

33 Wegen der Umständlichkeit des Verfahrens kam diese Regelung nie zum Tragen, so dass diese Regelung nicht fortgeschrieben wurde. 
Abb. 3: Auszüge aus der Rahmenvereinbarung über die Berufsschule; Abschnitte 6, 7 und 8: Stundentafeln und Lehrpläne, Abschlüsse sowie weitere schulische Berechtigungen

6. Unterrichtsumfang, Lehrpläne und Stundentafeln der Berufsschule

6.1 Der Unterricht der Berufsschule umfasst mindestens 12 Wochenstunden. Er besteht aus berufsbezogenem und allgemeinem Unterricht. Für den Unterricht gelten die von dem jeweiligen Land erlassenen Lehrpläne und Stundentafeln.

6.2 Der berufsbezogene Unterricht umfasst in der Regel 8 Wochenstunden. Er richtet sich nach den von der Kultusministerkonferenz beschlossenen Rahmenlehrplänen, die nach dem Gemeinsamen Ergebnisprotokoll vom 30. Mai 1972 mit den entsprechenden Ausbildungsordnungen abgestimmt sind.

6.3 Der Unterricht in der Berufsschule soll den Fremdsprachenunterricht entsprechend seiner Bedeutung für den jeweiligen Ausbildungsberuf angemessen berücksichtigen.6.4 Die Stundentafeln können neben einem Pflichtbereich einen Wahlpflichtbereich und/oder Wahlbereich vorsehen. Dabei können Wahlpflicht- und/oder Wahlfächer zur Stützung, Vertiefung und Erweiterung (z. B. Fremdsprachen) angeboten werden.

7. Abschlïsse und Zeugnisse der Berufsschule

7.1 Die Berufsschule führt zu einem eigenständigen Abschluss; er umfasst berufliche und allgemeine Qualifikationen. Die Berufsschule kann mit einer Abschlussprüfung abschließen.

7.3 Die Erteilung von Abschluss- und Abgangszeugnissen sowie der nachträgliche Erwerb des Abschlusszeugnisses richten sich nach der „Vereinbarung über den Abschluss der Berufsschule“ (Beschluss der Kultusministerkonferenz vom 01.06.1979).

8. Weitere schulische Berechtigungen

8.1 Im Abschlusszeugnis der Berufsschule kann nach den Bestimmungen des jeweiligen Landes entsprechend der unter Ziffer 7.3 genannten „Vereinbarung über den Abschluss der Berufsschule" ein Bildungsstand bestätigt werden, der in seinen Berechtigungen dem Abschlusszeugnis der Hauptschule entspricht.

8.2 Nach den Bestimmungen des jeweiligen Landes kann der Abschluss der Berufsschule einen dem Realschulabschluss gleichwertigen Abschluss einschließen. Die gegenseitige Anerkennung dieser Berechtigung wird über eine Vereinbarung der Kultusministerkonferenz angestrebt.

Quelle: $K M K(2007$, S. 6ff.)

$\mathrm{Zu}$ den Lehrplänen für die Berufsschule gibt es in den „,frühen“ Berufsschulverordnungen keine Regelungen. Brandenburg übernahm bis 1993 Lehrpläne aus den Ländern Berlin, Nordrhein-Westfalen und Niedersachsen (vgl. MBJS 1995, S. 19). Sachsen schreibt zunächst per Erlass für die Berufsschule den Einsatz von Lehrplänen aus Baden-Württemberg vor. Thüringen führt per Er- 
lass Lehrpläne aus Rheinland-Pfalz ein. ${ }^{34}$ Diese Lehrpläne wurden im Rahmen der Neuordnungen von Ausbildungsberufen schrittweise durch landesspezifische Lehrpläne für den berufsbezogenen Bereich ersetzt. Zudem wurden Anfang der 1990er Jahre jeweils landesspezifische Lehrpläne für den allgemeinen (berufsübergreifenden) Bereich erarbeitet.

In der Rahmenvereinbarung fehlen vollständig Angaben zur Leistungsfeststellung in der Berufsschule. Wohl auch deshalb ist die Leistungsfeststellung in den Berufsschulverordnungen bemerkenswert unterschiedlich geregelt. Mecklenburg-Vorpommern und Sachsen machen sehr detaillierte Vorgaben; so werden in der sächsischen Berufsschulverordnung Art und Anzahl der Leistungsnachweise sowie deren Bewertung vorgegeben. In der schulartïbergreifenden Verordnung von Sachsen-Anhalt sind keine Regelungen zur Leistungsfeststellung an der Berufsschule enthalten, Thüringen sieht als einziges Land schulische Abschlussprüfungen und eine Klassenwiederholung vor, während Brandenburg außer in Sport und Fachpraxis nur auf schriftliche Arbeiten abstellt und die Details an Klassen- und Jahrgangskonferenzen delegiert. Diese sehr unterschiedliche Regelungsdichte in den Berufsschulverordnungen der ostdeutschen Länder ist symptomatisch für die Ausweisung von Leistungsfeststellungen in den Berufsschulverordnungen aller Länder. Und sie ist auch eines der Hindernisse bei der Einbeziehung berufsschulischer Leistungsfeststellungen in das Ergebnis der Berufsausbildungsabschlussprüfung bei den zuständigen Stellen.

Für die schulischen Berechtigungen, die in Verbindung mit dem erfolgreichen Besuch der Berufsschule erworben werden können, ergibt sich aus den Berufsschulverordnungen der Länder zumindest für Außenstehende ein unübersichtliches Bild: Mit dem erfolgreichen Abschluss der Teilzeitberufsschule kann in Brandenburg die Berufsbildungsreife, erweiterte Berufsbildungsreife oder die Fachoberschulreife, in Mecklenburg-Vorpommern der Hauptschul- oder der Realschulabschluss, in Sachsen der Qualifizierende Hauptschulabschluss oder der Qualifizierte berufliche Bildungsabschluss, ${ }^{35}$ in Sachsen-Anhalt der Realschulabschluss sowie in Thüringen der Hauptschuloder der Realschulabschluss erworben werden. Dabei werden unterschiedliche und z. T. sehr detaillierte Bestehens- und Ausgleichsregelungen hinsichtlich der Mindestnotendurchschnitte sowie für den mittleren Bildungsabschluss hinsichtlich des Fremdsprachenunterrichts formuliert.

34 Vorläufige Lehrplanhinweise und Lehrpläne für die Schulen in Thüringen. In: ABl. des Thüringer Kultusministeriums und des Thüringer Ministeriums für Wissenschaft und Kunst Nr. 7, 1991, S. 294.

35 In der sächsischen Berufsschulverordnung wurden diese Bezeichnungen aus der entsprechenden bayerischen Verordnung übernommen. 


\section{Fazit}

Aus dem Vergleich wird deutlich, dass die neugegründeten Länder jeweils entsprechend ihrer unterschiedlichen berufsschulpolitischen Auffassungen die beruflichen Schulen regelten. Der Plural muss hier verwendet werden, weil diese Ansätze für alle beruflichen Bildungsgänge in den Schulgesetzen konstitutiv sind. Es blieb der jeweils herrschenden schulrechtlichen Ländersicht vorbehalten, Struktur und z. T. auch die Inhalte zu bestimmen. Inwieweit der Schulgesetzentwurf des deutschen Juristentages eine Rolle spielte, kann nicht geklärt werden. Martini schlussfolgerte bezüglich der schnellen Verabschiedung der ersten Schulgesetze in Thüringen, Brandenburg, Sachsen und Mecklenburg-Vorpommern im März bzw. April 1991, dass keine Grundsatzdiskussionen geführt wurden bzw. beabsichtigt waren (vgl. Martini 1992, S. 8). Allerdings gesteht sie zu, dass zur Herstellung von Rechtssicherheit schnell juristische Grundlagen geschaffen werden mussten (vgl. ebd.) Insofern gab es hier eine der für diese Untersuchung nachgefragten „Zwangsläufigkeiten“. ${ }^{36}$ Daraus ergibt sich, dass die darauf beruhenden Rechtsverordnungen, im vorliegenden Fall die Berufsschulverordnungen, auch jeweils diesen unterschiedlichen Sichtweisen folgten.

Mit Bezug auf die KMK-Rahmenvereinbarung über die Berufsschule kann festgestellt werden, dass in den Berufsschulverordnungen dem sog. "Gleichheitsminimum" weitgehend gefolgt wird, aber eine sehr große Bandbreite der Regelungen festzustellen ist. Alle Länder übernahmen weitgehend die Terminologie der KMK-Rahmenvereinbarung zur Gliederung in Grundund Fachstufe, zur Fachklassenbildung sowie zur Unterrichtsorganisation. Das BGJ wird überall als mögliche Grundstufe genannt, obwohl sich diese Organisationsform von Ausnahmen abgesehen in den westdeutschen Ländern gegen den Vorbehalt der Wirtschaft nicht durchgesetzt werden konnte. In den ostdeutschen Ländern wurde mit dem Angebot eines BGJ die Hoffnung ver-

36 Anhand der öffentlich zugänglichen Parlamentsdrucksachen ab der ersten Legislaturperiode, lässt sich für das Land Brandenburg eine breite berufsschulpolitische Diskussion feststellen (vgl. Hölterhoff S. 84) Die Details aus dem sächsischen Landtag sind wegen Nichtöffentlichkeit der Ausschussprotokolle nicht nachvollziehbar. In der Einbringungsrede kommen die Begriff „Berufsschule ${ }^{\text {“ bzw. „Berufliche Schulen }}$ " nicht vor (vgl. http://edas. landtag.sachsen.de/ 1. Legislaturperiode, Stand 2.12.2014) Im thüringischen Landtag kam der Begriff „Berufsschule“ in der 77. Sitzung bei der ersten Beratung des Schulgesetzes nur einmal bei der Aufzählung der Anzahl der Schulen vor (vgl. http://www.parldok.thueringen. de/ParlDok/dokument/8501/77-plenarsitzung.pdf\#page=12, Stand 2.12.2014) Im Landtag Mecklenburg-Vorpommern wurde bei der Einbringung des Schulreformgesetzes von Minister Oswald Wutzke ein Hinweis zur Doppelqualifikation bzw. Berufsausbildung mit Abitur gegeben (vgl. Plenarprotokoll 1/11 vom 27.2.1991, S. 380 f.). In der dritten Lesung am 25. April 1991 gab es keine Änderungsanträge zu den beruflichen Schulen. (vgl. Plenarprotokoll $1 / 19$, S. 783 f.) Der sachsen-anhaltinische Landtag befasste sich in der ersten Lesung nicht mit Fragen der beruflichen Schulen. (vgl. Plenarprotokoll 1/12 S.624 f.). 
knüpft, durch ein vollzeitschulisches erstes Ausbildungsjahr die Verweildauer in der Berufsausbildung zu verkürzen und damit einen Beitrag zur Entspannung der zunächst schwierigen Ausbildungsplatzsituation leisten zu können. Dafür spricht auch das Angebot eines kooperativen BGJ in den Schulgesetzen der Länder Brandenburg und Thüringen. Insofern lässt sich feststellen, dass der Transformationsprozess in diesem Bereich weitestgehend aus der unreflektierten Übernahme bisheriger Regelungen bestand. ${ }^{37}$

Unterschiedliche Vorgehensweisen und Regelungen sind in den Berufsschulverordnungen immer dann festzustellen, wenn dazu keine konsensualen Lösungen (z. B. für die Leistungsfeststellung in der Berufsschule) in der Rahmenvereinbarung angeboten bzw. enthalten sind oder wenn in der Rahmenvereinbarung nur der „kleinste gemeinsame Nenner“" gefunden wurde. Ein Bespiel dafür sind die weiteren schulischen Berechtigungen, die an der Berufsschule erworben werden können. Sowohl die jeweils landesspezifisch gewählten Benennungen als auch jeweils unterschiedlichen landesspezifischen Maßstäbe für den Erwerb dieser Berechtigungen erschweren den Vergleich. Dieser spezifische Sachverhalt deutet im Übrigen darauf hin, dass es in den 1990er Jahren durchaus noch Vorbehalte gegen die Gleichwertigkeit schulischer Abschlüsse beruflicher und allgemeiner Bildung gab.

Insgesamt zeigen die Berufsschulverordnungen jedoch, dass die Rahmenvereinbarung für die neugegründeten Länder orientierend und - mit Einschränkungen - auch normativ wirkte. Dabei bildet sich die Rahmenvereinbarung nicht nur in den Berufsschulverordnungen, sondern auch in einigen Regelungen der Schulgesetze sowie in stärkerem Maße in einschlägigen Verwaltungsvorschriften ab. Die Einschränkungen lassen sich wie folgt erklären: Aus dem langen Entstehungsprozess der Rahmenvereinbarung ist abzuleiten, dass sich die ,alten“ Länder lediglich über einen Minimalkonsens verständigt haben. Auf der Suche nach Orientierung war dieser Minimalkonsens für die neugegründeten Länder hilfreich, aber nicht ausreichend. Somit kann aus dem Transformationsprozess gefolgert werden, dass die Rahmenvereinbarung nur in begrenztem Rahmen zielführend war.

Abschließend soll an dem Beispiel der Leistungsfeststellung in der Berufsschule deutlich gemacht werden, dass die Vereinheitlichung von sechzehn Regelungen oder weiter gefasst, eine einheitliche Regelung durch die Länder nicht möglich war und sein wird. Die berufsschulpolitische und auch berufsbildungspolitische Debatte zwischen dem Bund und den Ländern kulminierte gegen Ende der 1990er Jahre in Zusammenhang mit der Weiterentwicklung der beruflichen Bildung am Beispiel der Anrechnung der berufsschulischen Leistungsbewertung bei der Berufsausbildungsabschlussprüfung vor der zuständigen Stelle. So forderte Ossenbühl (vgl. Ossenbühl 2001) in

37 Diese Untersuchung kann noch keine Aussagen treffen, ob in den Partnerländern ebenso wie in den anderen westdeutschen Ländern bereits der 12 Wochenstunden umfassende Unterricht vorgeschrieben war. 
seinem Rechtsgutachten als Voraussetzung dafür ein Mindestmaß an Vergleichbarkeit bei der Leistungsfeststellung in der Berufsschule (Gleichheitsminimum) durch entsprechende Regelungen in den Ländern, die zudem über einen Länderstaatsvertrag zu gewährleisten sind. Der Vergleich der ostdeutschen einschlägigen Schulverordnungen zeigt, wie weit derzeit noch der Weg bis zu einer gleichwertigen Leistungsfeststellung in der Berufsschule ist.

An diesem Beispiel wird deutlich, dass die föderalistische Struktur im Bildungssystem, die letztlich auf Freiwilligkeit beruht und sich im Wesentlichen bewährt hat, eine rechtförmliche Verbindlichkeit nur durch eine staatsvertragliche Grundlage für die KMK und den daraus ableitbaren rechtsverbindlichen, d. h. in Landesrecht umzusetzenden Beschlüssen herstellen könnte.

Vergleiche zu Unterschieden und Gemeinsamkeiten von DDR-Regelungen zur Berufsschule und einschlägigen KMK-Rahmenvereinbarungen sowie den Berufsschulverordnungen der westdeutschen Länder wurden vorerst nicht durchgeführt. Deshalb ist keine Aussage möglich, welche Brüche die Umstellung auf das neue Recht jenseits politischer Vorgaben bewirkte.

\section{Literatur}

Akademie (1983). Akademie der Pädagogischen Wissenschaften der Deutschen Demokratischen Republik (Hrsg.), Das Bildungswesen der Deutschen Demokratischen Republik. (2. Aufl.). Berlin.

Ernst, U. (2014). Die Entwicklung der Gymnasien bzw. gymnasialen Oberstufen in Brandenburg seit 1990. In: C. Ritzi \& F. Tosch (Hrsg.), Gymnasium im strukturellen Wandel - Befunde und Perspektiven von den preußischen Reformen bis zur Reform der gymnasialen Oberstufe (S. 203-245). Bad Heilbrunn: Klinkhardt.

Fuchs, H.-W. (1997). Bildung und Wissenschaft seit der Wende. Zur Transformation des ostdeutschen Bildungssystems. Opladen: Springer Verlag.

Hölterhoff, D. (2014). Berufsbildende Schulen in den ostdeutschen Ländern am Neuanfang. Paderborn: Eusl.

Less, H. (1949). Die Berufsausbildung an der Schwelle des zweiten Planjahres. Berufsbildung, 315, 4-7.

Jehkul, W., Belkner, P. \& Allmann,V. (1991). Erstes Schulreformgesetz für das Land Brandenburg. Textsammlung und Kommentar. Gütersloh.

KMK (Hrsg.) (1990). Berufliches Schulwesen in der Zuständigkeit der Länder. Dokumentation eines Seminars am 6./7. September 1990. Bonn (unveröffentlicht).

KMK (Hrsg.) (1998). Einheit in der Vielfalt. 50 Jahre Kultusministerkonferenz 1948 1998. Neuwied.

KMK (Hrsg.) (2007). Zusammenfassende Darstellung einschlägiger Beschlüsse der Kultusministerkonferenz. Bonn.

Kuklinski, P. \& Wehrmeister, F. (1998). Die Re-Kommunalisierung der DDR-Berufsschule. Recht der Jugend und des Bildungswesens, 1, 99-109. 
Lüdtke, M. \& Quast, H. (1992). Die Rolle der Treuhandanstalt bei der Entwicklung der beruflichen Bildung in den neuen Bundesländern. In: B. Seyfried \& P. Wordelmann (Hrsg.), Neue Länder - Neue Berufsausbildung? Berufsschulgesetz der DDR von 1990 und IGBBiG von 1990 - Proze $\beta$, Probleme und Perspektiven des Übergangs der Berufsausbildung in den neuen Bundesländern. Berichte zur beruflichen Bildung, 153, 135-149. Berlin.

Martini, R. (1992). Schullandschaft im Umbruch. Gesetzliche Regelungen für das allgemeinbildende Schulwesen in den neuen Bundesländern. Frankfurt am Main: Deutsches Institut für Internationale Pädagogische Forschung.

MBJS (Hrsg.) (1995). OSZ-Bericht 95. Die Oberstufenzentren im Land Brandenburg. Stand und Perspektiven der Entwicklung. Potsdam.

Ossenbühl, F. (2001). Rechtsgutachten zur Einbeziehung berufsschulischer Leistungsfeststellungen in die Berufsschule. Erstattet dem Bundesministerium für Bildung und Forschung. Bonn (unveröffentlicht).

Wehrmeister, F. (2005). Betriebsberufsschulen in der ehemaligen DDR. In: Klaus Illerhaus (Hrsg.): Festschrift anlässlich der 250. Sitzung des Unterausschuss für Berufliche Bildung der KMK (S. 70-85). Bonn. 


\section{Anlage 1: Rechtliche Regelungen}

Anordnung über die Förderung des Berufsnachwuchses in volkseigenen Betrieben (Rundschreiben der Deutschen Wirtschaftskommission und Deutschen Verwaltung für Volksbildung). In: Zentralverordnungsblatt Jg. 1948, Nr. 22 vom 6. Juli 1948, S. 260

Beschluss des Ministerrates der DDR über die Berufsausbildung der Lehrlinge in der sozialistischen Wirtschaft vom 28. Juni 1956. In: Dokumente zur Entwicklung des Schulwesens in der Deutschen Demokratischen Republik. Teil 2: 1956 bis 1967/68, 1. Halbband, Berlin 1969, S. 42-46

Direktive über die Berufsausbildung mit Abitur vom 6. Juli 1960. In: Verfügungen und Mitteilungen des Ministeriums für Volksbildung Jg. 1960 S.186 f.

Gesetz über die sozialistische Entwicklung des Schulwesens vom 2. Dez. 1959. In: Dokumente zur Entwicklung des Schulwesens in der DDR. Teil 2: 1956 bis 1967/68, I. Halbband, Berlin 1969, S. 42-46

Gesetz über das einheitliche sozialistische Bildungssystem vom 25. Febr. 1965. GB1. der DDR I 1965 Nr. 6 S.83

Gesetz über die Inkraftsetzung des Berufsbildungsgesetzes der Bundesrepublik Deutschland in der Deutschen Demokratischen Republik (IGBBiG) vom 19. Juli 1990. GBl. der DDR I Nr. 50 S. 907 (veröff. am 13.08.1990)

Gesetz über Berufsschulen vom 19. Juli 1990. GBl. der DDR I Nr. 50 S. 919 (veröff. am 13.08.1990)

Gesetz- und Verordnungsblatt Thüringen: Nr. 5, S. 61, Vorläufiges Bildungsgesetz (VBiG) vom 25. März 1991

Gesetz- und Verordnungsblatt für das Land Brandenburg Teil I-Nr. 16 vom 8. Juli 1992: Erstes Schulreformgesetz für das Land Brandenburg (Vorschaltgesetz - 1. SRG) vom 28. Mai 1991

Gesetz-und Verordnungsblatt Land Sachsen-Anhalt: Nr. 17/199 vom 17. 7.1991:

Schulreformgesetz für das Land Sachsen-Anhalt (Vorschaltgesetz) vom 11. Juli 1991

Gesetz-und Verordnungsblatt für den Freistaat Sachsen (SächsGVB1.: S. 213): Schulgesetz für den Freistaat Sachsen (SchulG) vom 3. Juli 1991

KMK (1959): Abkommen über das Sekretariat der Ständigen Konferenz der Kultusminister der Länder in der Bundesrepublik Deutschland vom 20. Juni 1959

KMK (1991): Rahmenvereinbarung über die Berufsschule. Beschluss der Kultusministerkonferenz vom 15.03.1991. Beschlusssammlung der KMK - Berufliche Bildung, Beschluss Nr. 323. Loseblattsammlung, Neuwied 
KMK (1998): Hohenheimer Memorandum zur Wissenschafts-, Bildungs- und Kulturpolitik im geeinten Deutschland. Mitteilungen und Informationen des Sekretariats der Kultusministerkonferenz, Nr. 252, 25.02.1991, Mitteilungen und Informationen

Landtag von Sachsen-Anhalt: - Erste Wahlperiode - Plenarprotokoll 1/1 6 - 24. 05. 1991 S. 975

Landtag Mecklenburg-Vorpommern:, Plenarprotokoll 1/11 vom 27.2.1991 S. 374 f. und Plenarprotokoll vom 1/19 vom 25.4.1991, S. $783 \mathrm{f}$

Landtag Mecklenburg-Vorpommern: Entwurf eines Ersten Schulreformgesetzes. Drucksache 1/144 vom 13.2.1991.

Thüringer Schulordnung für die Berufsschule (ThürBSO) v. 10. Dezember 1996 AB1 Nr. 2/1997 v. 16. Januar 1997, S. 33 - 39

Verfassungsgesetz zur Bildung von Ländern in der Deutschen Demokratischen Republik (Ländereinführungsgesetz) vom 22. Juli 1990. GBl. DDR 1990 I S. 955

Verordnung über die Ausbildung von Industriearbeitern in den Berufsschulen (In Kraft getreten am 3. Nov. 1947). In: Berufsbildung 2 (1948) 1, S. 28-32

Verordnung über die Verantwortung und die Aufgaben bei der Leitung der Berufsbildung. In: Sozialistisches Bildungsrecht. Hrsg. vom Staatssekretariat für Berufsbildung, Berlin (Ost) 1985, S. 33-42

Verordnung über die Facharbeiterberufe - Systematik der Ausbildungsberufe vom 21. Dez. 1984. GBl. I 1985 Nr. 4 S. 28

Verordnung über die Aufrechterhaltung von Leistungen betrieblicher Kindergärten, polytechnischer und berufsbildenden Einrichtungen vom 6. Juni 1990. GB1. der DDR I Nr. 32 S. 297 (veröff. am 19.06. 1990)

Verordnung über Berufsbildende Schulen (BbS-VO) v. 11. Juni 1992/GVBl LSA Nr. 24/1992 v. 17. Juni 1992, S. $441-463$

Verordnung des Sächsischen Staatsministeriums für Kultus (SMK) über die Berufsschule (Schulordnung Berufsschule - BSO) vom 11. März 1994. GVB1. S. 477

Verordnung über die Berufsschule in Mecklenburg-Vorpommern (BSVO) vom 5. Juli 1996/ GVOBl 1996 M-V S. 480

Vertrag über die Schaffung einer Währungs-, Wirtschafts- und Sozialunion zwischen der Bundesrepublik Deutschland und der Deutschen Demokratischen Republik vom 18. Mai 1990. BGB1. 1990 II S. 537

Vertrag zwischen der Bundesrepublik Deutschland und der Deutschen Demokratischen Republik über die Herstellung der Einheit Deutschlands (Einigungsvertrag) vom 31. Aug. 1990. BGBl.1990 II S. 889

Vorläufige Berufsschulordnung im Land Brandenburg (BSO) vom 7. Januar 1992/ GVB1. II Nr. 7 v. 18. Febr. 1992, S. 40-45 


\title{
Anlage 2: Verzeichnis der Abkürzungen
}

\author{
BBA Berufsbildungsausschuss \\ BBiG Berufsbildungsgesetz \\ BBS Betriebsberufsschule \\ BFS Berufsfachschule \\ BGJ Berufsgrundbildungsjahr \\ BIBB Bundesinstitut für Berufsbildung \\ BSZ Berufliches Schulzentrum \\ BVJ Berufsvorbereitungsjahr \\ BVJA Berufsvorbereitungsjahr für Ausländer und Aussiedler \\ BVJE Berufsvorbereitungsjahr für Erwachsene \\ BVJS schulisches Berufsvorbereitungsjahr \\ DDR Deutsche Demokratische Republik \\ F1, F2 Förderlehrgänge des Arbeitsamtes gemäß SGB III/AFG 40 c 4 \\ FOS Fachoberschule \\ FS Fachschule \\ GAL Grundausbildungslehrgänge gemäß SGB III/AFG 40 c 4 \\ HwO Handwerksordnung \\ JOA Schüler (Jugendliche) ohne Ausbildungsvertrag \\ KBS kommunale Berufsschule \\ KMK Ständige Konferenz der Kultusminister der Länder in der Bundesrepublik \\ Deutschland \\ LAB Landesausschuss für Berufsbildung \\ OSZ Oberstufenzentrum \\ PrA Prüfungsausschuss gemäß BBiG/HwO \\ SRG Erstes Schulreformgesetz Brandenburg \\ StSchA Staatliches Schulamt (Brandenburg) \\ $\mathrm{UABBi}$ Unterausschuss für Berufliche Bildung der $\mathrm{KMK}$
}

